\title{
Spinal Epidural Cavernous Hemangioma: Fluctuating Symptom
}

\author{
Woo Man Jeon, Sung Hwa Paeng, Won Hee Lee, Young Woo Shim, Sung Tae Kim \\ Department of Neurosurgery, Inje University Busan Paik Hospital, Inje University School of Medicine, Busan, \\ Republic of Korea
}

Corresponding author:

Sung Hwa Paeng

Department of Neurosurgery,

Medicine, Inje University

Busan Paik Hospital, Inje

University School of Medicine,

Bokji-ro 75, Busangjin-gu,

Busan 47392, Republic of Korea

Tel: +82-51-890-6144

Fax: +82-51-898-4244

E-mail: shpaeng@empas.com

Received: May 12, 2020

Revised: June 10, 2020

Accepted: June 29, 2020

\begin{abstract}
Although spinal epidural cavernous hemangioma $(\mathrm{CH})$ is rare, identified cases have been increasing in number. We report a case of spinal epidural $\mathrm{CH}$ with a fluctuating symptom and discuss its diagnostic evaluation and treatment. A 24-year-old woman had left shoulder pain and mild sensorimotor impairment of the T6 dermatome 5 months before. On examination for pathological reflex, the deep tendon reflex was found to be somewhat elevated. No abnormalities were observed in a simple radiological evaluation. The results of the electrophysiological evaluations were normal. The patient's symptoms improved after medication and physical therapy. After 3 months, she developed a sudden-onset paraparesis (2/5) and sensory change below her nipple. Magnetic resonance imaging revealed a well-defined extradural mass at the thoracic level. An emergency surgical resection was performed. After the surgery, immediate recovery of the sensorimotor response in the lower extremity was attained. The pathological finding was $\mathrm{CH}$. Fluctuating sensorimotor symptoms should be carefully evaluated immediately because their etiology is involved in the hemodynamic changes in $\mathrm{CH}$.
\end{abstract}

Key Words: Epidural space; Hemangioma, cavernous, central nervous system; Hematoma, epidural, spinal; Paraparesis

\section{INTRODUCTION}

Benign vascular lesions located primarily in the extradural space within the spine are uncommon. They constitute $<6 \%$ of all spinal neoplasms and are represented classically by cavernous hemangioma $(\mathrm{CH})$, angiolipoma, and arteriovenous hemangioma $^{8)}$. These vascular malformations may be found in any part of the neuraxis. The most frequent type is a $\mathrm{CH}$, which generally presents as single or multiple intracranial lesions or as lesions arising within the vertebral bodies from where they may extend secondarily into the extradural space. The aim of this case report was to describe an uncommon fluctuating neurological symptom caused by spinal epidural $\mathrm{CH}$.

\section{CASE REPORT}

A 24-year-old woman presented with a 5-month history of left shoulder pain and mild sensorimotor impairment of the T6 dermatome. On examination for pathological reflex, the deep tendon reflex was found to be elevated. The basic radiological and cranial nerve neurological evaluation results were normal. Her somatosensory evoked potential, electro-myogra- phy result, and nerve conduction velocity were normal. Her symptoms improved after medication and physical therapy, and she returned to her activities in daily living. After 3 months, her condition suddenly deteriorated. Neurological examinations revealed sudden-onset paraparesis (2/5) and sensory change below the nipple. Thoracic lumbar magnetic resonance (MR) imaging revealed a large left epidural mass extending from the T2-T3 level to the T4-T5 level and compression of the adjacent thecal sac, with displacement to the right side (Fig. 1A, B). The lesion showed hyperintensity on T2-weighted images (WIs), isointensity on T1-WIs, and slightly heterogeneous enhancement (especially at the upper and lower portions of the mass) on Gd-T1-WIs. The lesion extended through the left neural foramen at T3-T4 into the paraspinal area (Fig. 1C, D). An emergency surgical resection was performed. Under general anesthesia, the patient underwent hemilaminectomy. No attachment to the nerves or dura was found. The mass was soft and reddish, with severe bleeding. Preserved planes were found all around. We performed total resection of the mass. The pathological finding was $\mathrm{CH}$ (Fig. 2). The lesion was composed of multiple, dilated, and ectatic vascular channels containing blood. After the total surgical resection, immediate recovery of the sensorimotor response in the lower extremity was attained. The patient had no symptoms at 1-year follow-up. 


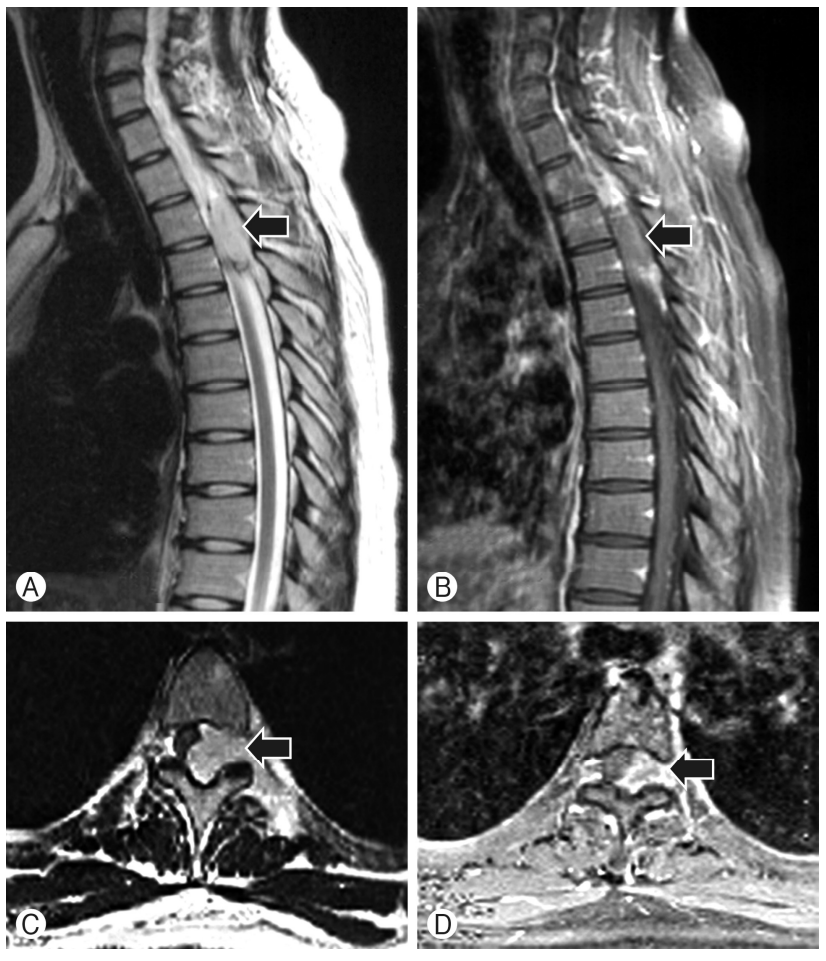

Fig. 1. T-spine magnetic resonance (MR) imaging scan shows large epidural mass extending from T2-3 to T4-5, pushing the spinal cord to right side. This lesion (black arrow) shows slightly high signal intensity on sagittal T2-weighted image (WI) (A), and slightly heterogeneous enhancement (especially upper and lower portion of mass) on sagittal gadolinium-enhanced T1-WI(B). (C) Axial T2-wighted MR image showing high signal intensity lesion that this extends through the left neural foramen of T3-4 into the paraspinal area (black arrow). (D) Axial gadolinium-enhanced T1-weighted MR image at the same level shows intense and heterogeneous enhancement of the extradural lesion (black arrow).

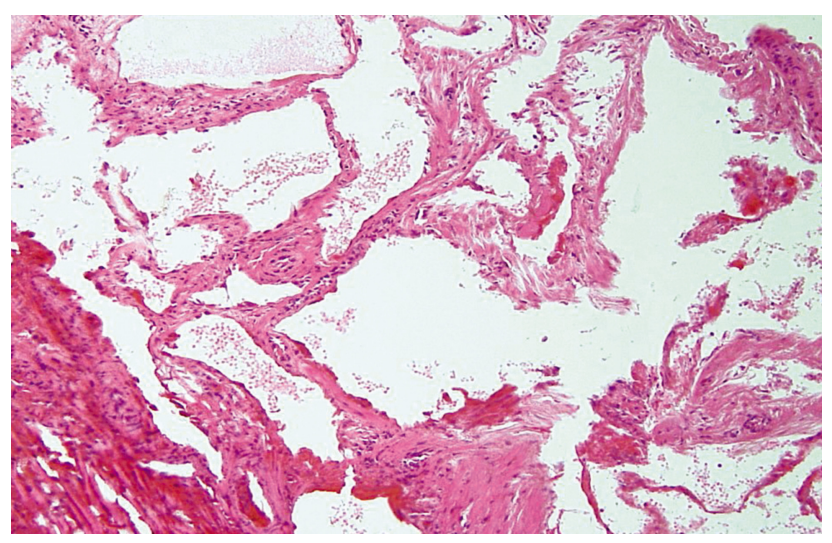

Fig. 2. Photomicrograph shows the typical findings of cavernous hemangioma. Well-circumscribed cavernous hemangioma composed of thin-walled sinusoidal vascular spaces of varying sizes lined with a single layer of endothelial cells. Elastic and muscular laminae were not seen (hematoxylin and eosin stain [H\&E], ×200 magnification).

\section{DISCUSSION}

Purely extradural cavernous or arteriovenous hemangioma is rare, accounting for only $<4 \%$ of all extradural spinal tumors ${ }^{7}$. Although it has no mitotic activity, it grows slowly, probably because of recurrent hemorrhage and thrombotic phenomenon with organization and recanalization ${ }^{8}$, manifesting as spinal cord syndrome, local pain (neck and back), and radiculopathy.

The clinical course is usually slowly progressive. Acute clinical deterioration may be observed in a few cases in relation to the significant growth of the lesion, intralesional hemorrhage, or thrombotic venous occlusion ${ }^{6}$. The propensity to bleed is explicated by the thin-walled vessels and stasis of blood flow in the lesion ${ }^{2)}$. Other causes of acute symptoms include estrogen-mediated neoangiogenesis in the lesion. The spinal cord is fragile to sudden-onset massive compression due to hemodynamic changes (e.g., bleeding) (Fig. 3). Symptoms occurring with these hemodynamic changes are referred to as fluctuating symptoms.

CHs are nearly identical at any location throughout the body. Its pathological composition includes irregular sinusoidal vascular channels with thin collagenous walls lined by a single layer of flattened endothelium. Elastic and smooth muscle fibers within the walls are scant if not absent. At the thoracic level, they contain a higher proportion of fat. Extradural CHs show a paucity of interstitial hemosiderin deposits in comparison with intraparenchymal lesions ${ }^{2)}$.

Extradural CHs show high signal intensity on T2-weighted MR images, which may be explained by their high content of stagnant blood. Slow blood flow may contribute substantially to the signal, conferring a low or intermediate signal intensity on T1-WIs. The MR differential diagnosis of extradural hemangioma also includes sequestrated herniated disk, extradural

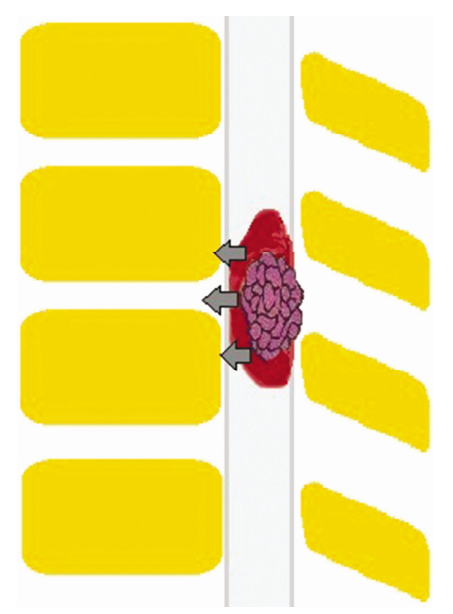

Fig. 3. This illustration showing that spinal cord is fragile to sudden onset massive compression due to hemodynamic change. 
nerve sheath tumor, epidural infiltrating disease, epidural abscess, and epidural cyst. Especially in cases of dumbbell-shaped hemangioma, the differential diagnosis includes schwannoma or neurofibroma ${ }^{3,7)}$. The morphological characteristics of extradural hemangiomas described in the literature ${ }^{3)}$ and as seen in our cases include the round or ovoid shape of the lesion and its tendency to extend through the intervertebral foramen. Angiography is not useful for diagnosis because of extradural $\mathrm{CH}$ has no communication with spinal cord arteries.

Epidural hemangiomas show no tendency to regress, and complete resection of the lesion seems to be the best treatment, as it relieves patients of symptoms and provides a good prognosis in most cases.

Aoyagi et al. ${ }^{1)}$ reported that the diagnosis is almost always missed on preoperative evaluation. Therefore, although the lesion is rare and a high index of suspicion is required for the exact diagnosis based on preoperative imaging, $\mathrm{CH}$ should be included in the differential diagnosis of purely extradural soft tissue lesions of the spine.

In young females, the first symptom was slight but recurring. This case shows the tendency of the symptoms to recur, which makes it unique compared with other cases ${ }^{4,5,9)}$.

\section{CONCLUSION}

Early and accurate diagnosis followed by complete excision of the lesion prior to any possible episodes of intralesional bleeding is related to a favorable outcome. Sudden intralesional hemorrhage can lead to fluctuating symptoms due to spinal cord compression and subsequent severe disability.

\section{CONFLICTS OF INTEREST}

No potential conflict of interest relevant to this article was reported.

\section{REFERENCES}

1. Aoyagi N, Kojima K, Kasai H: Review of spinal epidural cavernous hemangioma. Neurol Med Chir (Tokyo) 43:471-475, 2003

2. Feider HK, Yuille DL: An epidural cavernous hemangioma of the spine. AJNR Am J Neuroradiol 12:243-244, 1991

3. Harrington JF, Jr., Khan A, Grunnet M: Spinal epidural cavernous angioma presenting as a lumbar radiculopathy with analysis of magnetic resonance imaging characteristics: Case report. Neurosurgery 36:581-584, 1995

4. Jang D, Kim C, Lee SJ, Ryu YJ, Kim J: Pure spinal epidural cavernous hemangioma with intralesional hemorrhage: A rare cause of thoracic myelopathy. Korean J Spine 11:85-88, 2014

5. Li TY, Xu YL, Yang J, Wang J, Wang GH: Primary spinal epidural cavernous hemangioma: Clinical features and surgical outcome in 14 cases. J Neurosurg Spine 22:39-46, 2015

6. Padovani R, Poppi M, Pozzati E, Tognetti F, Querzola C: Spinal epidural hemangiomas. Spine (Phila Pa 1976) 6:336-340, 1981

7. Padovani R, Tognetti F, Proietti D, Pozzati E, Servadei F: Extrathecal cavernous hemangioma. Surg Neurol 18:463-465, 1982

8. Rovira A, Rovira A, Capellades J, Zauner M, Bella R, Rovira M: Lumbar extradural hemangiomas: Report of three cases. AJNR Am J Neuroradiol 20:27-31, 1999

9. Sanghvi D, Munshi M, Kulkarni B, Kumar A: Dorsal spinal epidural cavernous hemangioma. J Craniovertebr Junction Spine 1: 122-125, 2010 